\title{
Benefits of Using a Mars Forward Strategy for Lunar Surface Systems
}

\author{
Jack Mulqueen $^{1}$ \\ Marshall Space Flight Center, MSFC, Alabama 35812 \\ Brand Griffin ${ }^{2}$ \\ Marshall Space Flight Center, MSFC, Alabama 35812 \\ David Smitherman ${ }^{3}$ \\ Marshall Space Flight Center, MSFC, Alabama 35812 \\ and \\ Dauphne Maples ${ }^{4}$ \\ Marshall Space Flight Center, MSFC, Alabama 35812
}

\begin{abstract}
This paper identifies potential risk reduction, cost savings and programmatic procurement benefits of a "Mars Forward" Lunar Surface System architecture that provides commonality or evolutionary development paths for lunar surface system elements applicable to Mars surface systems. The objective of this paper is to identify the potential benefits for incorporating a Mars Forward development strategy into the planned Project Constellation Lunar Surface System Architecture. The benefits include cost savings, technology readiness, and design validation of systems that would be applicable to lunar and Mars surface systems. The paper presents a survey of previous lunar and Mars surface systems design concepts and provides an assessment of previous conclusions concerning those systems in light of the current Project Constellation Exploration Architectures. The operational requirements for current Project Constellation lunar and Mars surface system elements are compared and evaluated to identify the potential risk reduction strategies that build on lunar surface systems to reduce the technical and programmatic risks for Mars exploration. Risk reduction for rapidly evolving technologies is achieved through systematic evolution of technologies and components based on Moore's Law superimposed on the typical NASA systems engineering project development "V-cycle" described in NASA NPR 7120.5. Risk reduction for established or slowly evolving technologies is achieved through a process called the "Mars-Ready Platform" strategy in which incremental improvements lead from the initial lunar surface system components to "Mars-Ready" technologies. The potential programmatic benefits of the Mars Forward strategy are provided in terms of the transition from the lunar exploration campaign to the Mars exploration campaign. By utilizing a sequential combined procurement strategy for lunar and Mars exploration surface systems, the overall budget wedges for exploration systems are reduced and the costly technological development gap between the lunar and Mars programs can be eliminated. This provides a sustained level of technological competitiveness as well as maintaining a stable engineering and manufacturing capability throughout the entire duration of Project Constellation.
\end{abstract}

\footnotetext{
${ }^{1}$ Aerospace Engineer, MSFC Advanced Concepts Office/ED04

${ }^{2}$ Aerospace Engineer, MSFC Advanced Concepts Office/ED04

${ }^{3}$ Space Architect, MSFC Advanced Concepts Office/ED04

${ }^{4}$ Systems Engineer, MSFC Advanced Concepts Office/ED04
} 


\section{Introduction}

The objective of this paper is to present a strategy to develop lunar and Mars surface system infrastructure elements for future human lunar and Mars Exploration. This strategy is called "Mars Forward" because it is based on a technology development approach, and possibly even more importantly, a budgetary procurement approach that provides for the development of lunar mission surface system architectures while maintaining a clearly defined and integrated path for evolving system elements for applications at Mars. This strategy is more than merely defining common systems. It is a sequential approach for developing surface system architecture elements that takes into account the different rates of technology development as well as the different requirements for lunar and Mars surface systems.

\section{Literature Search of Lunar and Mars Surface Systems}

The study began with a survey of previous human lunar and Mars exploration studies. Figures 1 and 2 illustrate the timelines, spanning over fifty years, of previous studies and reports on human exploration of the moon and Mars. Many of these studies were reviewed for this study to identify characteristics and general requirements that have been identified for lunar and Mars surface systems. The Marshall Space Flight Center's Advanced Concepts Office has developed an extensive electronic library of the most significant human lunar and Mars studies, dating back to the 1960's. These documents were systematically reviewed to identify information related to surface system design. The information included descriptions of surface system habitats, mobility systems, extravehicular activities, and the use of in-situ resources. Design and technology information was gathered on subsystems such as power, thermal control, communications and life support. It was found that even though most of the studies focused on the transportation systems rather than surface systems, specific information on surface system designs and requirements was found in 26 studies. Despite the fact that the studies reviewed spanned almost 50 years, basically covering the range from the Apollo era to the present time, there is remarkable consistency in the description of the surface systems and the technological requirements to develop self-sustaining surface system architectures on the moon or Mars. This consistency is the foundation of the Mars Forward strategy.

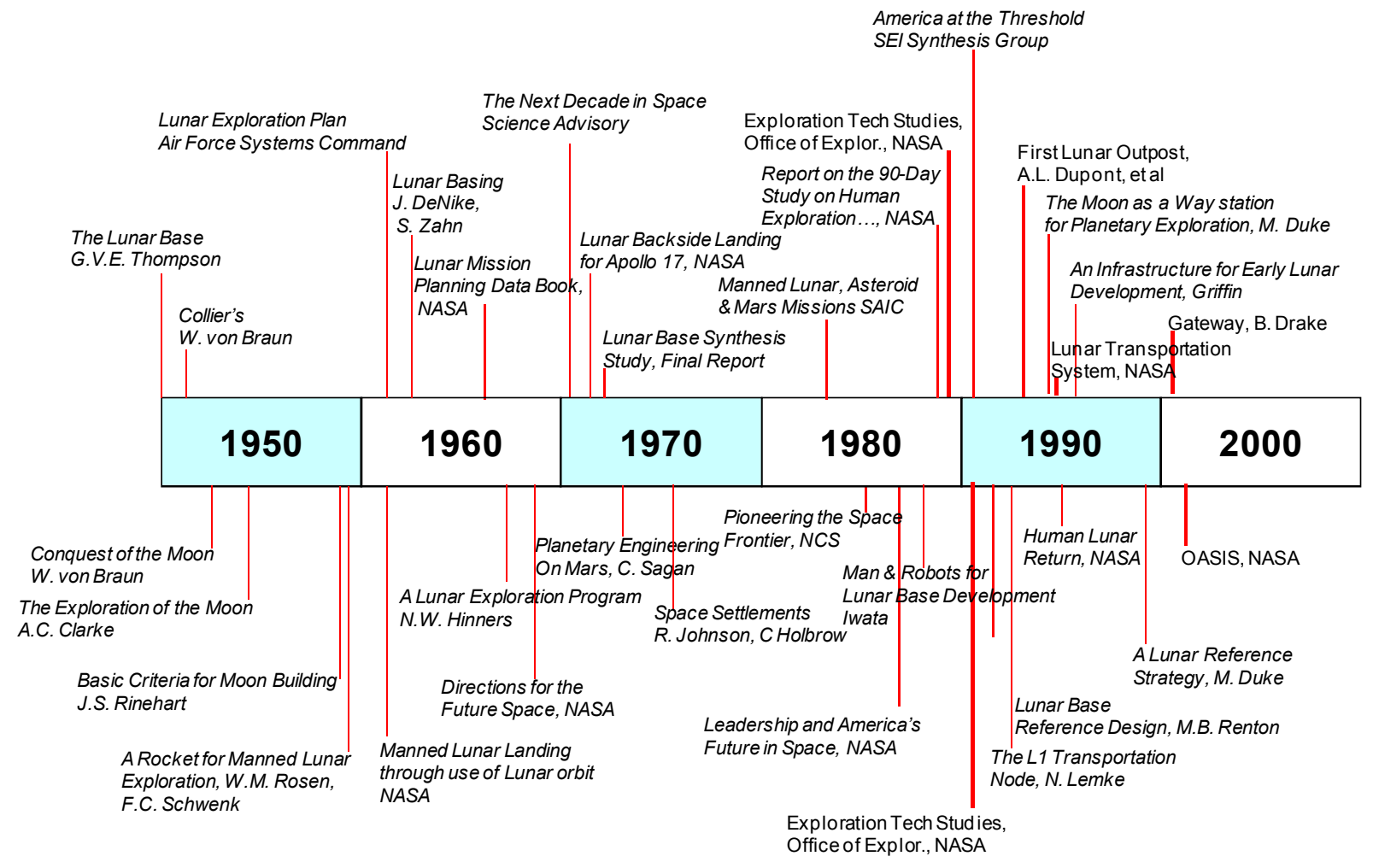

Figure 1. Timeline for Lunar Studies. 


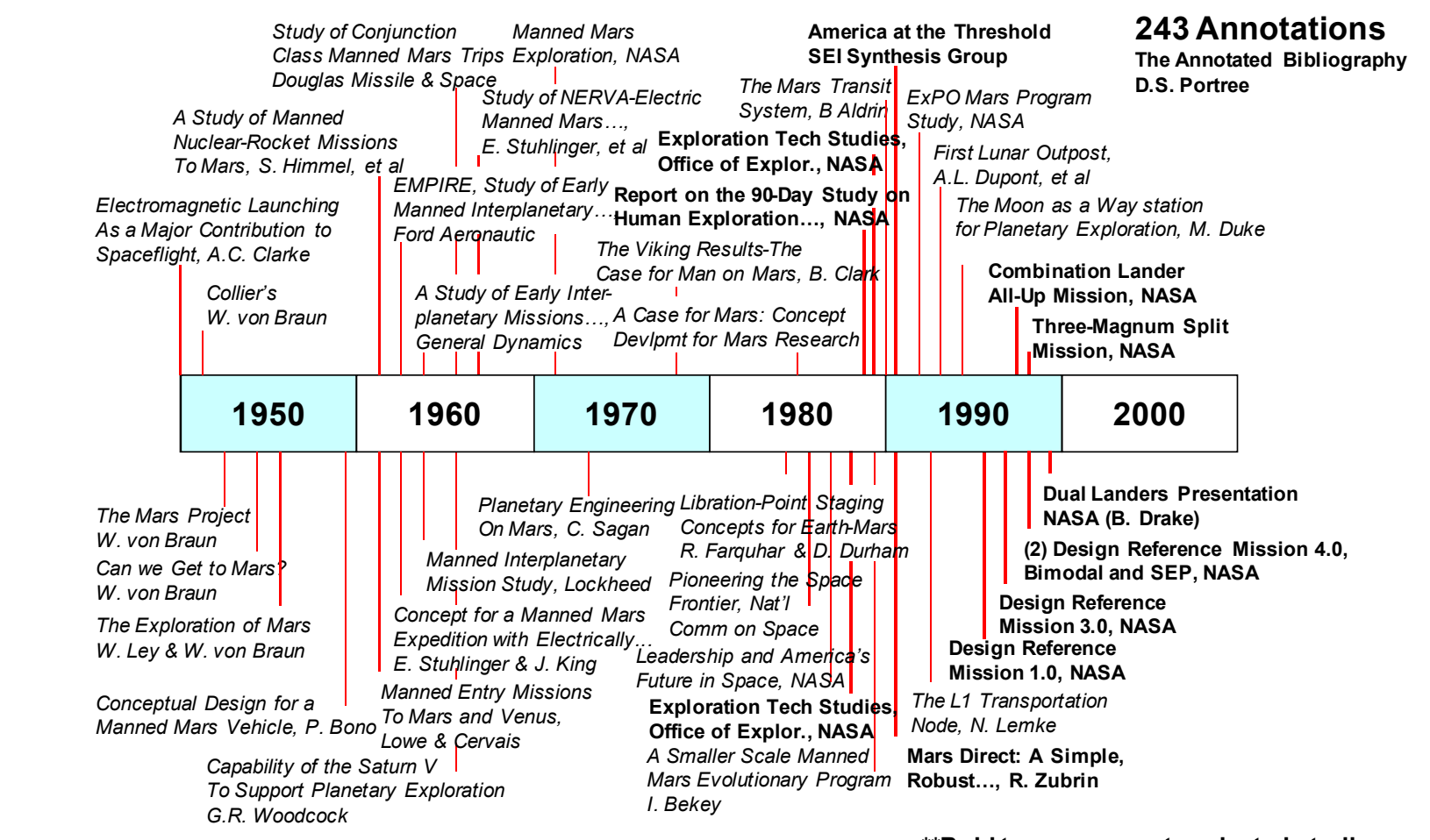

*A Comparison of Transportation Systems for Human Missions to Mars, AIAA $20043834 \quad$ **Bold type represents selected studies

Figure 2. Timeline for Mars Studies.

\section{Mars Forward Strategy}

The goal of implementing a Mars Forward strategy for the development of surface system elements is to provide cost and risk reduction for lunar and Mars exploration surface systems. The potential cost savings can be achieved by eliminating the gap of five or more years between the end of the lunar program procurements and the beginning of the Mars program procurements. This gap is shown graphically in the top diagram of Figure 3. It is likely that a gap of this duration would result in decrease of corporate experience, engineering continuity, manufacturing capability and possibly commercial competitiveness. Many of the start-up activities associated with the lunar program would have to be repeated for the Mars program at a higher cost. The Mars Forward strategy would employ a combined procurement strategy to meet the requirements for both lunar and Mars programs. This procurement approach would provide a program to build up to the level required to meet the goals of the lunar program and yet maintain engineering and manufacturing continuity to build up the required systems to meet the needs of the Mars program. Since this strategy takes advantage of the initial procurement investments for the lunar program, the magnitude of manufacturing investments for Mars surface systems may actually taper off as shown in the lower diagram of Figure 3. 


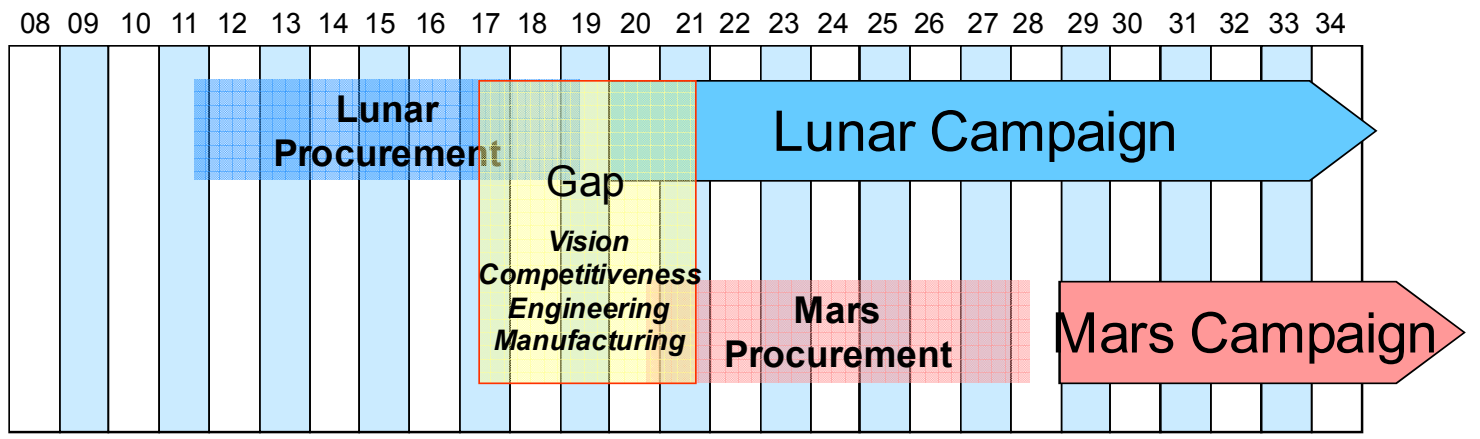

\section{Combined Procurement}

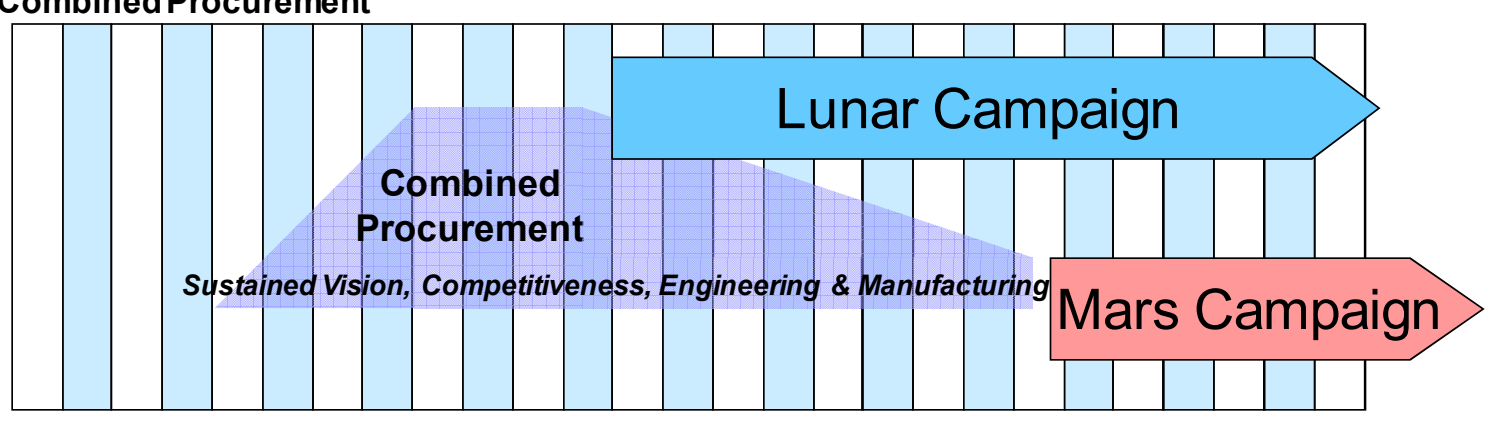

Figure 3. Procurement Approaches for Lunar and Mars Programs

Using the Mars Forward strategy, the lunar systems would be developed to provide direct risk reduction benefits for the Mars program. This can be illustrated by examining elements of the current Project Constellation. Three areas of potential commonality between lunar and Mars surface systems are shown in Figure 4. Both programs include an integrated surface habitat/lander, pressurized rovers and unpressurized rovers. With proper design early in the development cycle these systems could be developed to common requirements such that the lunar program provides a path to evolve from lunar applications to Mars applications. This forward investment is illustrated in Figure 5, using the unpressurized rover as an example. Rather than develop the rover for the 1/6 gravity of the moon, the basic structure of the rover would be developed for the 3/8 gravity of Mars. Rover subsystems such as the suspension system could be tuned for either environment. By making a relatively small investment to impose additional requirements on the development of the lunar rover, the Mars program would have lower cost, shorter delivery schedule and most importantly reduced risk because the lunar rover would become a "Mars Ready" surface architecture element. 
Figure 4. Candidates for Mars Ready Platforms.

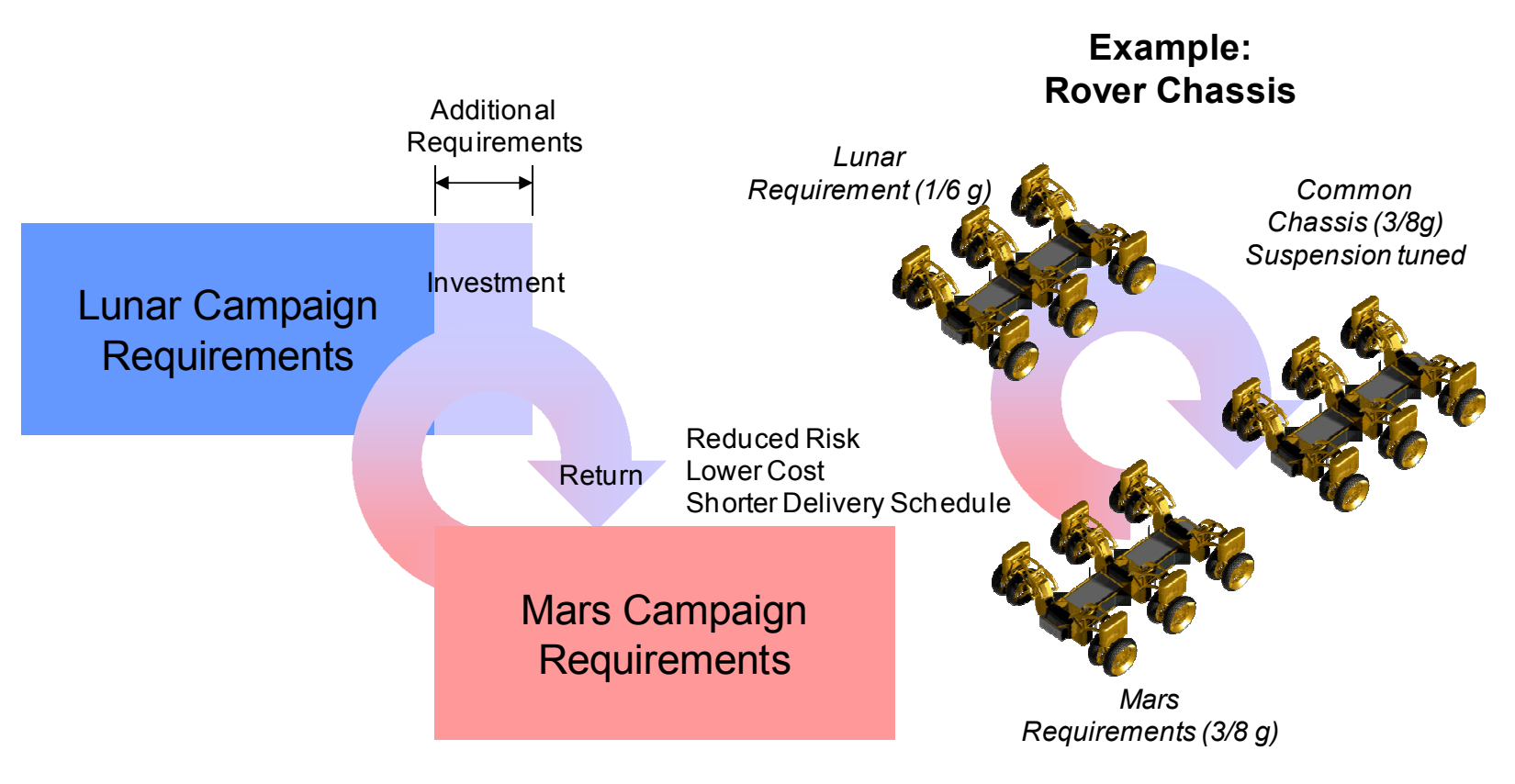

\section{Example: \\ Rover Chassis}

CxAT Lunar Campaign

CxAT Lunar Campaign

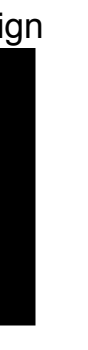

Higue Candidates for Mars Ready Platforms.
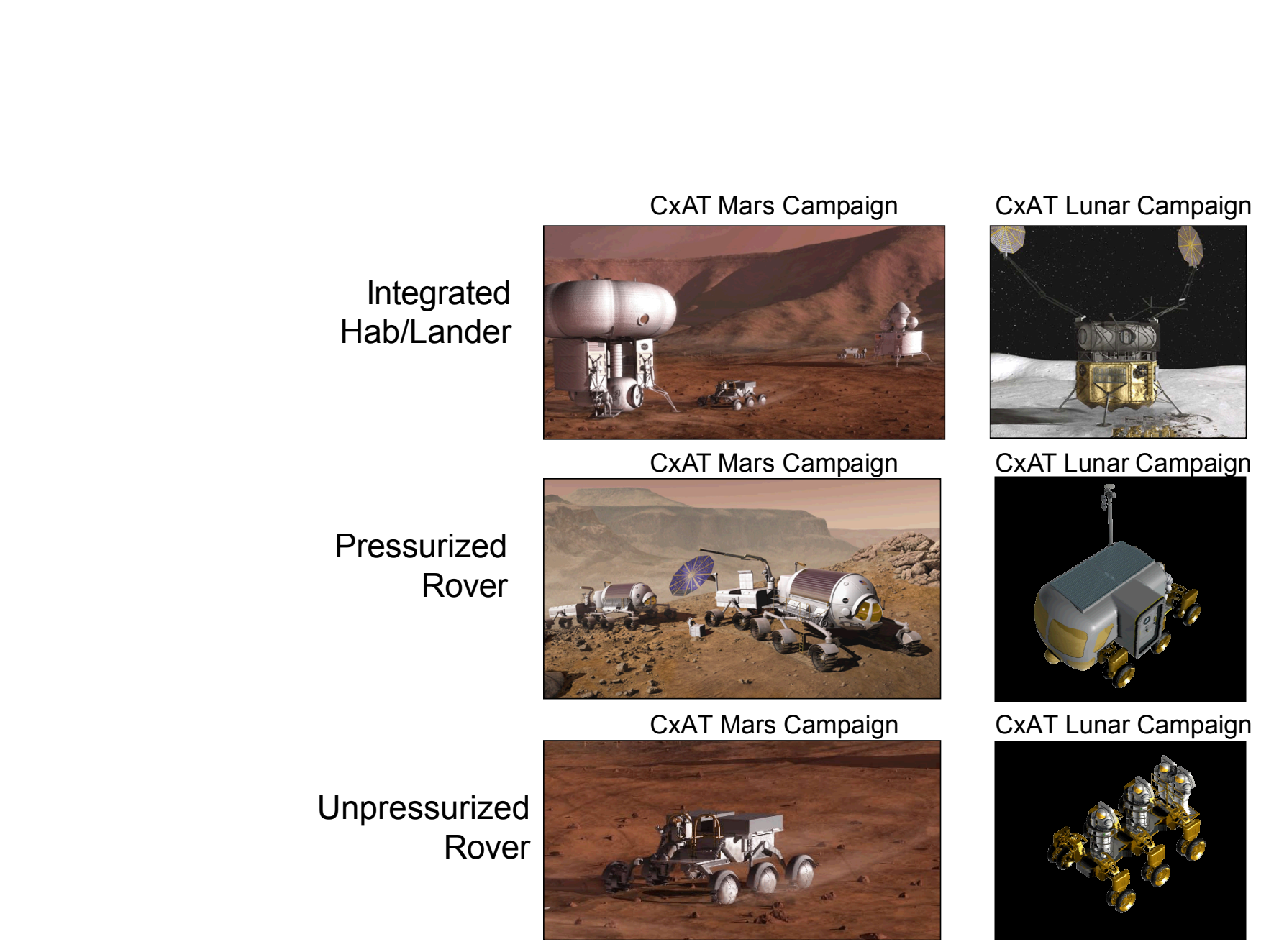

Figure 5. Mars Forward Investment and Return. 


\section{Mars Ready Platforms}

The proposed use of the lunar program to provide "Mars Ready" platforms to allow surface system evolution from lunar needs to Mars requirements is based on many similar examples in aerospace technology development. One of the best examples of the platform approach to system evolution is the B-52 aircraft shown in Figure 6. This aircraft has been in use since the 1950's and engineering analysis has shown it could be utilized for several decades into the future. The basic aircraft configuration has served as a platform that has undergone approximately 31 upgrade programs resulting in a modernized aircraft that meets requirements that did not even exist when the aircraft was initially developed. The evolution from lunar systems to Mars ready systems can be accomplished at many levels of system development ranging from integrated architecture elements, to the individual part level, as shown in Figure 7. The foundation technology platforms would be based on the technologies with relatively slow technology advancement, these platforms would evolve to Mars Ready systems with the implementation of rapidly developing technologies at the subsystem, component and part levels.

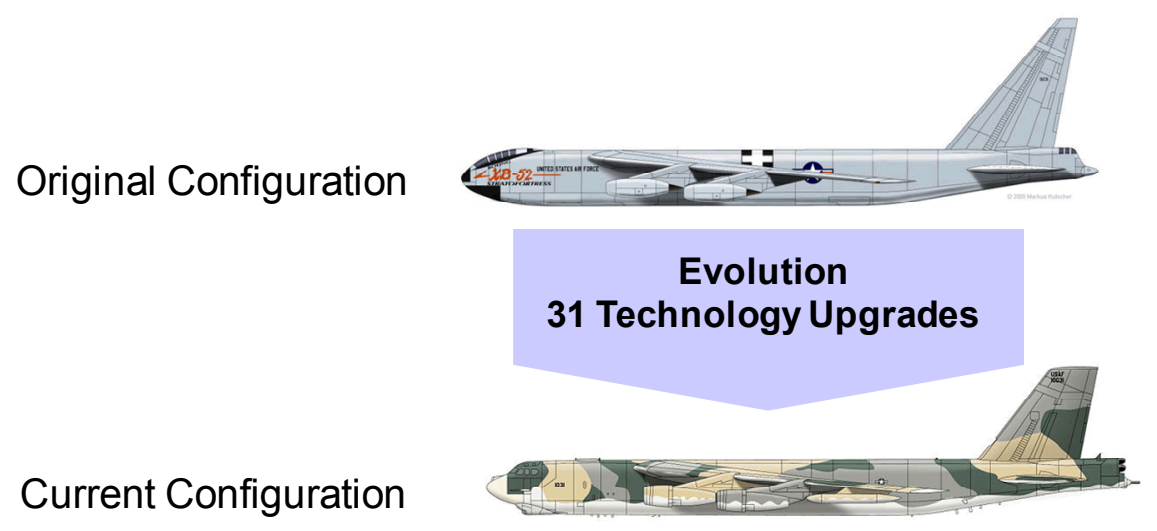

Source: Global Security.com, Weapons of Mass Destruction

Figure 6. B-52 Platform Evolution.

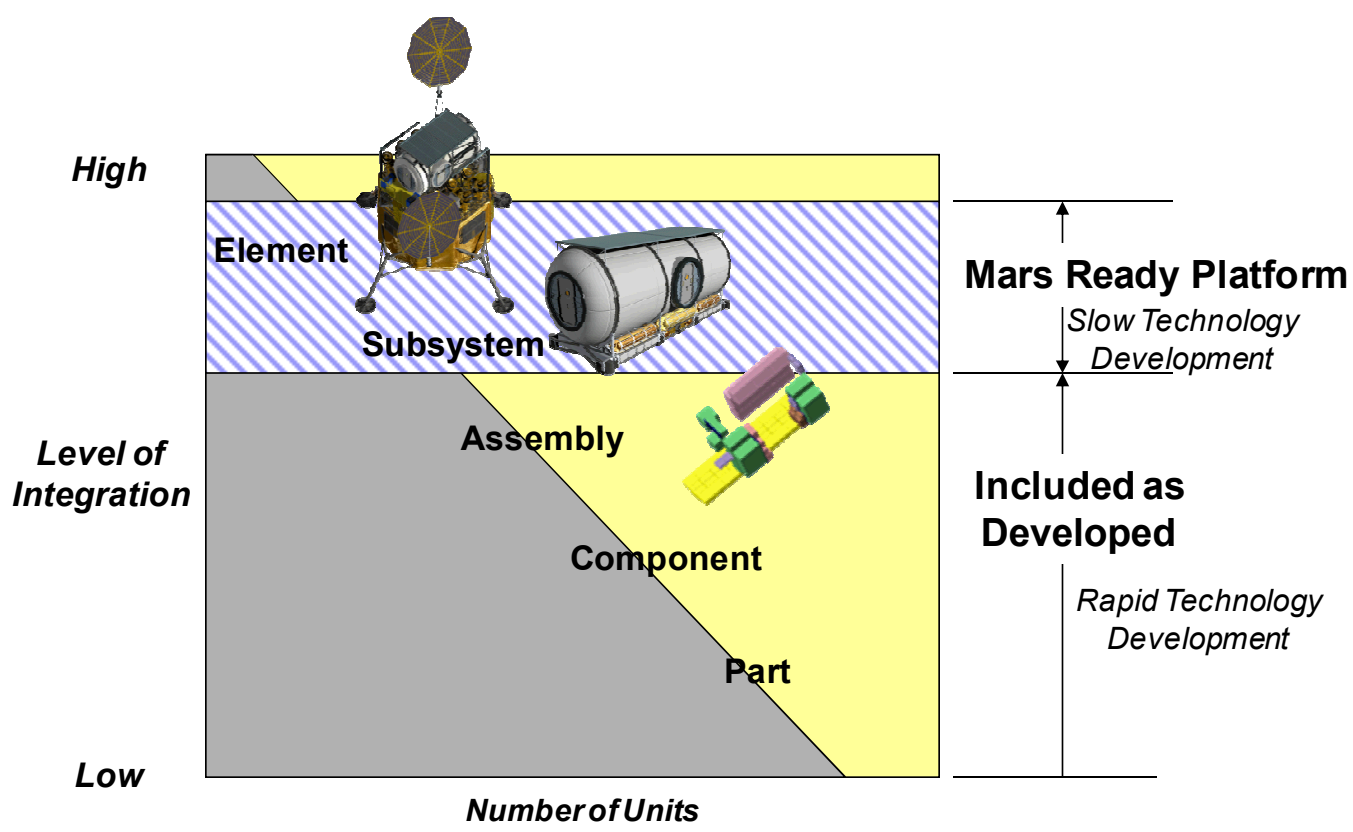

Figure 7. Mars Forward Technology Evolution Levels.

American Institute of Aeronautics and Astronautics 
An example of how incorporation of rapidly improving technology into a Mars Ready platform would benefit the Mars program is the area of crew resource automation. The evolution of a basic platform technology is evident in the pictures of the Boeing 707 and 787 aircraft cockpits shown in Figure 8. The original Boeing 707 aircraft required a four person crew for trans-Atlantic flights. Through the application of advanced avionics and resource automation in the design of the cockpit operations, the Boeing 787 requires only a two person crew. Current Project Constellation scenarios assume a crew of four for lunar surface exploration and a crew of six for Mars exploration. By assuming continued development of crew resource automation it should be possible to reduce the Mars crew size to four. This would allow commonality between the lunar and Mars programs. The lunar habitats, crew systems and life support systems could serve as platforms for evolving technologies needed for Mars.

\section{Aircraft Cockpit Resource Automation}
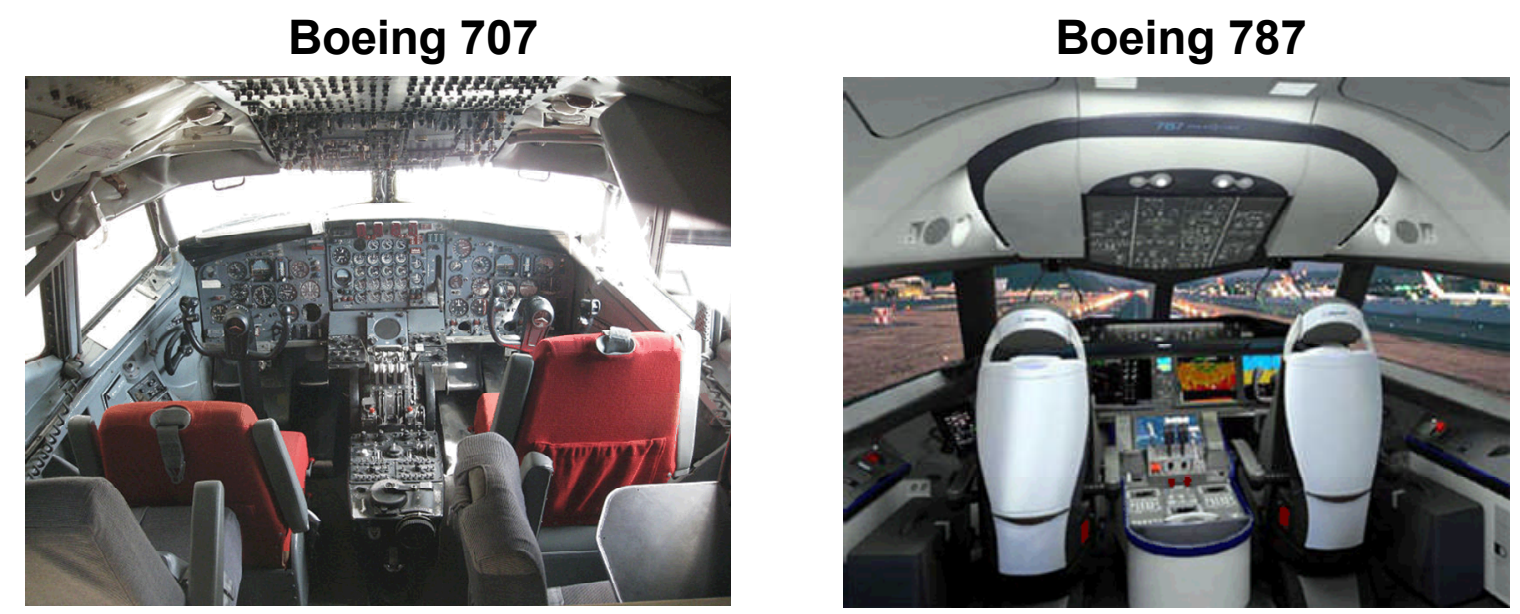

Figure 8. Aircraft Example of Technology Evolution.

\section{Benefits to Project Development Cycle}

NASA programs generally follow a cycle of development, fabrication, and integration verification as shown in the Project Cycle "V" diagram shown in Figure 9, from NASA Procedural Document, NPR 7120.5. In complex programs, each architecture element and system component undergoes its' own development cycle, resulting in multiple "V" cycles occurring simultaneously, but at different rates, all feeding into the integrated program. The technology platform approach to technology evolution allows a sequential technology development path that allows rapidly developing technologies, some of which may not even exist at the beginning of the lunar program, to be integrated into the standard NASA Project processes. These rapidly developing new technologies often follow Moore's law in which system performance doubles at a rapid rate, as has been the case with the number of transistors on an integrated circuit as shown in Figure 10. When superimposing or infusing these rapidly evolving technologies into the standard NASA program V cycle, programmatic efficiency is achieved through incremental development fabrication and integration of technologies resulting in enhanced functionality of existing systems. This process is illustrated in Figure 11, which shows how incremental technology evolution would benefit the lunar campaign and then feed forward into the Mars campaign. This enhanced functionality provides a direct evolutionary development path as well as risk reduction for meeting the Mars program requirements. 


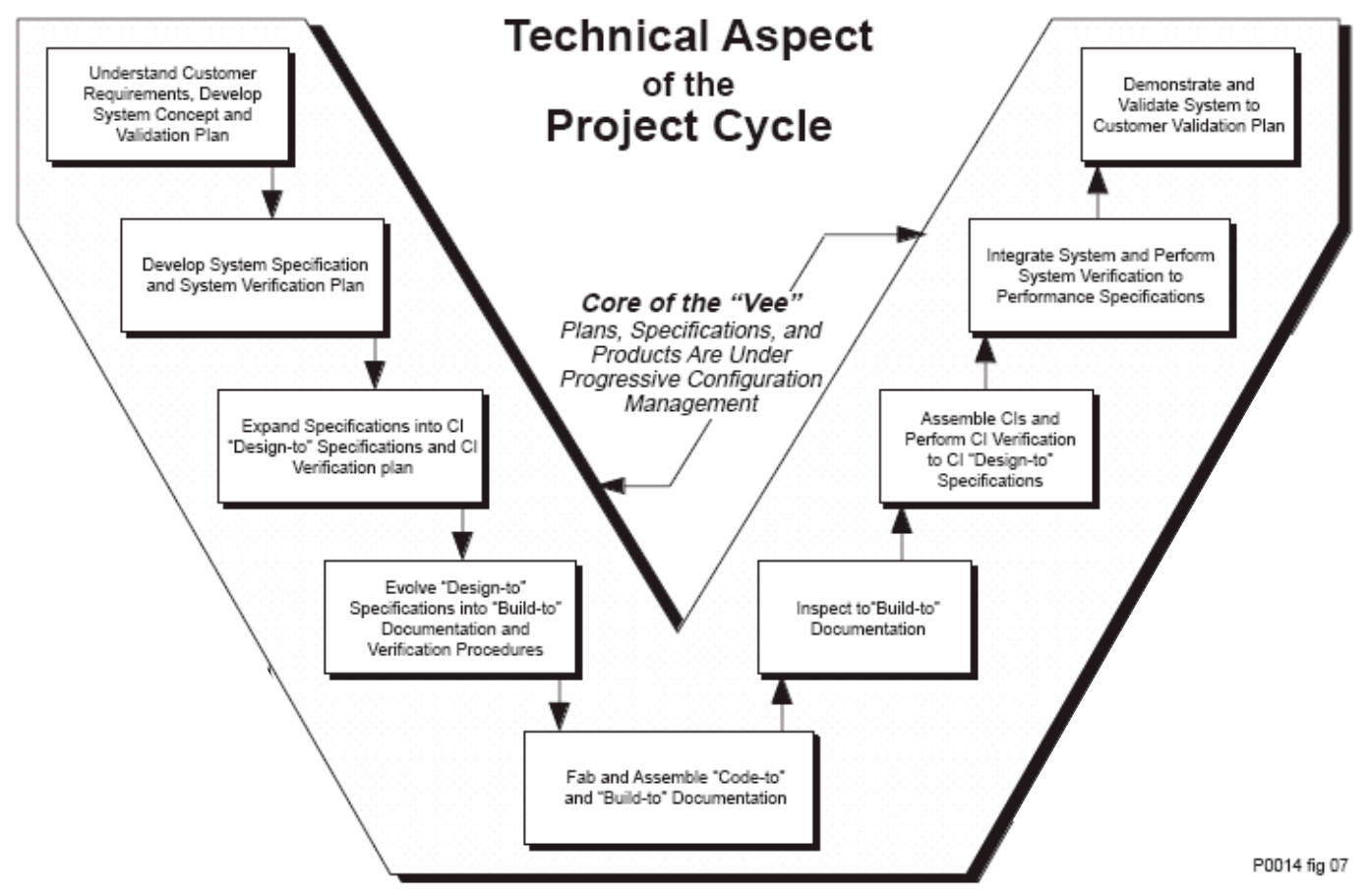

Figure 9. NASA NPR 7120.5 Project "V" Cycle.

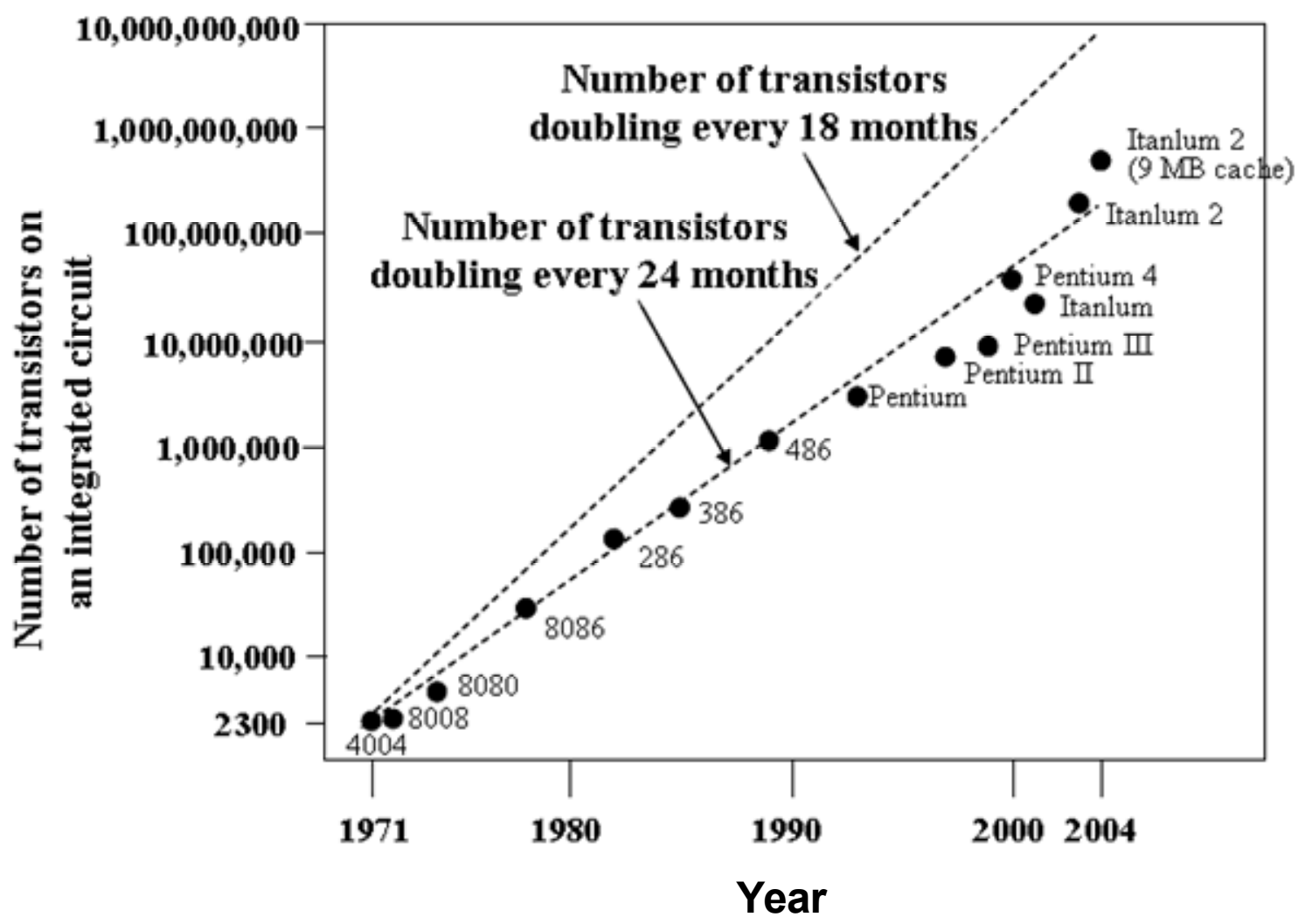

Figure 10. Moore's Law for Rapidly Improving Technologies 


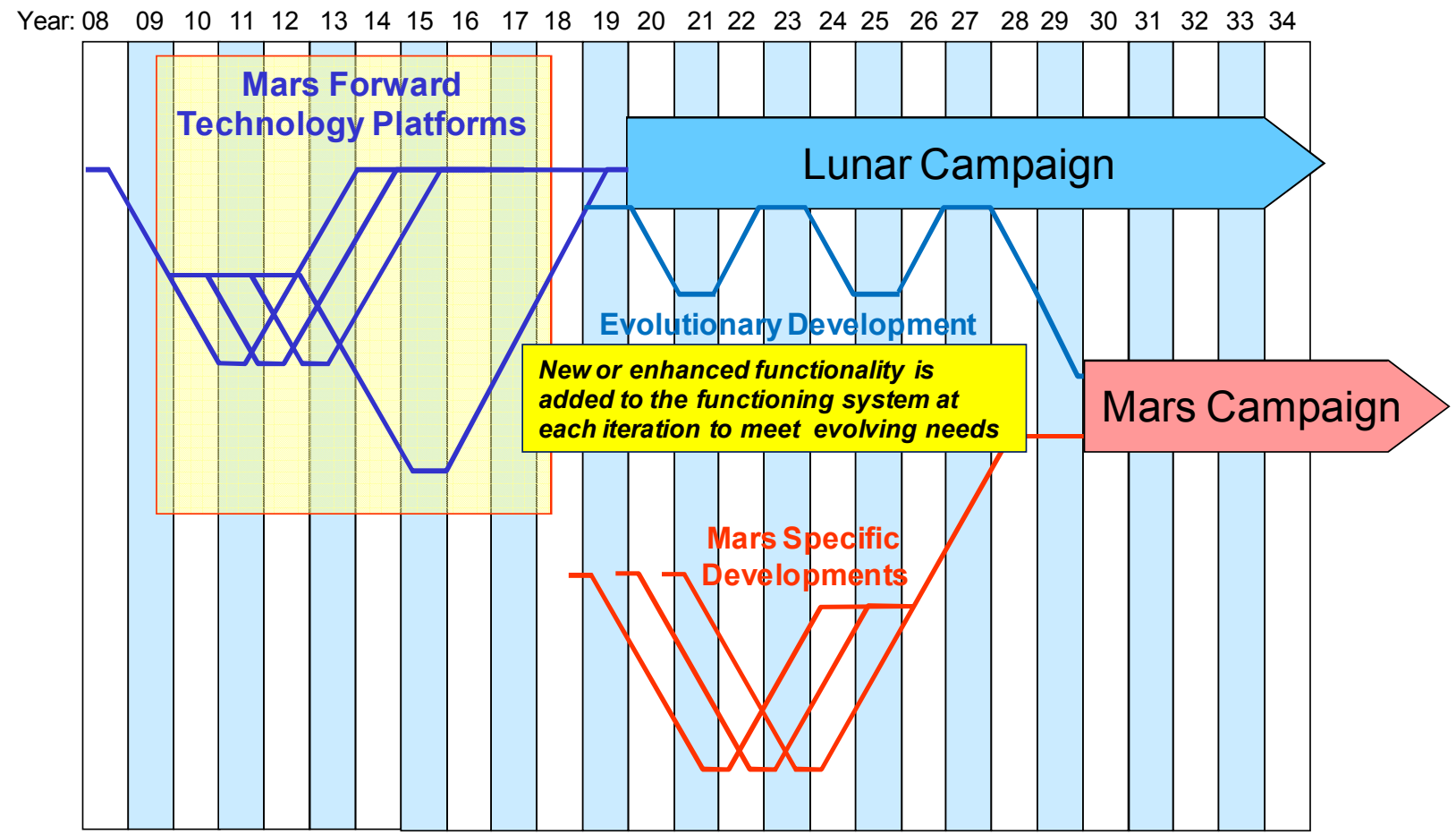

Figure 11. Evolutionary Technology Development Applied to Lunar and Mars Development Cycles.

\section{Conclusions}

A Mars Forward development strategy for developing lunar exploration surface systems provides a highly efficient approach to achieving the technology development requirements for the Project Constellation surface systems. The foundation of the Mars Forward strategy is the commonality between the lunar and Mars surface system functionality. The key to the Mars Forward approach is the development of Mars Ready Technology Platforms that provide sequential and continuous development cycles providing incremental technology evolution and system risk reduction to meet Mars exploration requirements. 